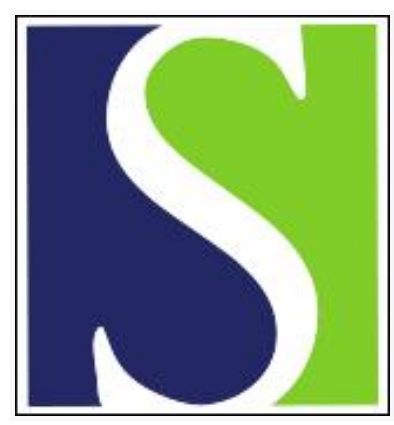

Scand J Work Environ Health 2003;29(2):143-151

https://doi.org/10.5271/sjweh.716

Issue date: Apr 2003

Neurobehavioral effects during experimental exposure to 1-octanol and isopropanol

by van Thriel C, Kiesswetter E, Blaszkewicz M, Golka K, Seeber A

Affiliation: Leibniz Research Centre for Working Environment and Human Factors, Ardeystrasse 67, 44139 Dortmund, Germany. thriel@ifade.de

The following articles refer to this text: 2004;30(6):486-496;

2009;35(1):1-5

Key terms: 1-octanol; alcohols; chemical sensitivity; distraction; experimental exposure; isopropanol; neurobehavioral effect; neuropsychology of attention

This article in PubMed: www.ncbi.nlm.nih.gov/pubmed/12718500 


\title{
Neurobehavioral effects during experimental exposure to 1-octanol and isopropanol
}

\author{
by Christoph van Thriel, PhD, ${ }^{1}$ Ernst Kiesswetter, PhD, ${ }^{1}$ Meinolf Blaszkewicz, PhD, ${ }^{1}$ Klaus Golka, MD, ${ }^{1}$ \\ Andreas Seeber, $P h D^{1}$
}

\begin{abstract}
van Thriel C, Kiesswetter E, Blaszkewicz M, Golka K, Seeber A. Neurobehavioral effects during experimental exposure to 1-octanol and isopropanol. Scand J Work Environ Health 2003;29(2):143-151.
\end{abstract}

\begin{abstract}
Objectives The study examined acute neurobehavioral effects provoked by controlled exposure to 1-octanol and isopropanol among male volunteers.

Methods In a 29- $\mathrm{m}^{3}$ exposure laboratory, 24 male students (mean age 25.8 years) were exposed to 1-octanol and isopropanol. Each substance was used in two concentrations ( 0.1 and 6.4 ppm for 1-octanol; 34.9 and 189.9 ppm for isopropanol:). In a crossover design, each subject was exposed for 4 hours to the conditions. Twelve subjects reported enhanced chemical sensitivity; the other 12 were age-matched controls. At the onset and end of the exposures neurobehavioral tests were administered and symptoms were rated.

Results At the end of the high and low isopropanol exposures the tiredness ratings were elevated, but no dosedependence could be confirmed. For both substances and concentrations, the annoyance ratings increased during the exposure, but only for isopropanol did the increase show a dose-response relation. The subjects reported olfactory symptoms during the exposure to the high isopropanol and both 1-octanol concentrations. Isopropanol provoked no sensory irritation, whereas high 1-octanol exposure slightly enhanced it. Only among the subjects with enhanced chemical sensitivity were both 1-octanol concentrations associated with a stronger increase in annoyance, and lower detection rates were observed in a divided attention task.

Conclusions Previous studies reporting no neurobehavioral effects for isopropanol (up to $400 \mathrm{ppm}$ ) were confirmed. The results obtained for 1-octanol lacked dose-dependency, and their evaluation is difficult. The annoying odor of 1-octanol may mask sensory irritation and prevent subjects with enhanced chemical sensitivity from concentrating on performance in a demanding task.
\end{abstract}

Key terms alcohols, chemical sensitivity, distraction, neuropsychology of attention.

Alcohols are neurotoxic substances that are widely used in industrial environments (eg, printing), as well as in hygiene (eg, disinfectants) and in household and personal care products. In household and personal care products alcohols with short carbon chains, like isopropanol, are used (1). Acute neurotoxic effects of isopropanol have been studied in controlled laboratory studies $(2,3)$, whereas acute and long-term effects of occupational exposure have been investigated in field studies (4-6). These studies have shown that even subjective signs of acute neurotoxicity like prenarcotic symptoms (4), sensory irritation (3), or impaired mood states (2) are unlikely during exposures up to
$400 \mathrm{ppm}$. Impaired performance in neurobehavioral tests was not found in studies investigating exposures in the range of current occupational exposure limits (2, 5). Thus the existing limits of $200 \mathrm{ppm}$ (Germany, MAK value) (7) and $400 \mathrm{ppm}$ (United States, ACGIH TLVTWA) (8) appear to be sufficient to prevent neurobehavioral effects.

However, exposures as high as the ones allowed by short-term exposure limits (STEL) of $400 \mathrm{ppm}$ (Germany) and $500 \mathrm{ppm}$ (United States) may cause acute effects associated with subjective symptoms of olfactory or trigeminal stimulation (eg, stench, eye irritation) and annoyance. Subjective symptoms and annoyance ratings

1 Leibniz Research Centre for Working Environment and Human Factors [Institut für Arbeitsphysiologie an der Universität Dortmund], Dortmund, Germany.

Corresponcence to: Dr Christoph van Thriel, Leibniz Research Centre for Working Environment and Human Factors, Ardeystrasse 67, 44139 Dortmund, Germany. [E-mail: thriel@ifado.de] 
are exposure effects with different components (9), namely, sensory and affective components. Intensity ratings of chemosensory symptoms (eg, irritation) are considered to reflect the sensory component of the stimulation; annoyance also covers an affective component associated with the consequences of the exposure, for example, feeling bothered or disturbed by the chemical.

Currently, alcohols with increasing carbon chain lengths (eg, 2-ethylhexanol, 1-octanol) are under consideration for limit setting, or threshold limit values (TLV) have recently been set. In Germany a MAK of $50 \mathrm{ppm}$ has been established for 2-ethylhexanol (10). Long-chain alcohols like 1-octanol and 2-ethylhexanol are used as drilling fluids (8), plasticizers, ingredients in perfumes and esters, and as a solvent and antifoaming agent (11). Workers who produce and handle such products (eg, floor covering) may be exposed to these chemicals, and neurotoxic effects are possible. Like short-chain alcohols, these volatile substances can enter the human organism by inhalation (10, 11 ), and they can be perceived via the chemical senses (12).

The authors of the German critical data evaluation for the MAK value of 2-ethylhexanol (10) complained about a lack of adequate human studies. Regarding 1octanol a similar dearth of human data exists. Only a few studies $(12,13)$ have examined the responses of human volunteers. The studies revealed decreasing odor thresholds and eye irritation with increasing carbon chain length (13). The same observation applies to the nasal pungency threshold (NPT) investigated in a group of anosmics (12). Whereas isopropanol has a nasal pungency threshold of $\approx 18135 \mathrm{ppm}$ (14), that of 1-octanol is only $\approx 80 \mathrm{ppm}$ (12). Consistent with the increasing trigeminal potency of $\mathrm{n}$-alcohols the anesthetic power, measured as the minimum alveolar anesthetic concentration (MAC), likewise increases with carbon chain length (15). Thus, compared with isopropanol, even lowlevel exposure to long-chain alcohols may provoke chemosensory or prenarcotic symptoms or performance deficits in neurobehavioral tests.

In order to ensure workers' safety and health, the subjective and behavioral effects of long-chain alcohols should be investigated (i) in humans and (ii) in exposure scenarios relevant for workplaces, including peak exposures as high as the respective short-term exposure limits. So that the special chemosensory qualities of 1octanol (eg, low odor and nasal pungency threshold) can be taken into account, human volunteers with heightened sensitivity towards chemicals or chemical odors should be included. Hypersensitivity to chemicals or their odors seems to be a growing problem even among young adults (16). Thus the purpose of our study was the prototypical comparison of isopropanol and 1-octanol as instances of short- and long-chain alcohols with respect to their neurobehavioral effects at different exposure levels in persons with different sensitivity responses to chemicals or chemical odors.

\section{Subjects and methods}

\section{Subjects}

One hundred and fifty male students were screened with a standardized questionnaire (17) on chemical and general environmental sensitivity. The questionnaire was composed of 67 statements concerning reactions to various environmental stimuli, and it served to determine self-reported chemical sensitivity. Specifically, self-reported chemical sensitivity was defined as clear agreement with at least one out of eight statements describing strong physical responses (eg, nausea) to chemicals (eg, lacquer vapor, gasoline). The scale used for the questionnaire is related to the concept of cacosmia (16). Comparable with that used with cacosmia questionnaires, it has served as a screening tool to identify chemically sensitive subjects in the general population. During the requirement process the participants were not informed of any details of the study design in relation to the data on chemical and general environmental sensitivity (eg, feedback of the individual score on the scale for self-reported chemical sensitivity).

On the basis of the screening, 15 chemically sensitive subjects and 16 controls were invited to participate in the study and examined by a physician. Subjects with diagnosed asthma, allergic rhinitis, psychiatric disorders, or chronic diseases (eg, diabetes, liver diseases) were excluded. The neuropsychological tests were repeatedly practiced to minimize learning effects during the course of the experiment. Finally, 24 healthy male students, mean age 25.83 (SD 4.26) years (12 chemically sensitive subjects and 12 age-matched controls), participated. None of the participants reported any type of olfactory impairment. Both groups showed comparable odor thresholds for butanol $\left(350 \mu \mathrm{g} / \mathrm{m}^{3}\right.$ for the chemically sensitive subjects and $341 \mu \mathrm{g} / \mathrm{m}^{3}$ for the controls). The local ethics committee approved the study protocol. Written informed consent was obtained from the subjects prior to the study.

\section{Exposure and procedure}

The experiments were carried out in an exposure laboratory at the Institute of Occupational Physiology at the University of Dortmund. The laboratory is a secluded room built of glass and stainless steel with spatial dimensions of $4.80 \times 2.65 \times 2.27 \mathrm{~m}\left(\approx 29 \mathrm{~m}^{3}\right)$. Inside the 
laboratory, four personal computer workstations were separated by three vertical boards. Four subjects were exposed simultaneously. Each workstation was equipped with a 15 -inch color computer monitor and various response panels for the neurobehavioral testing and computer-aided rating of subjective symptoms and annoyance.

From an adjacent room, a climate-control unit provided the laboratory with tempered and humidified air. During the exposures, the average relative humidity in the laboratory was about $40 \%$ with a mean temperature of $\approx 25^{\circ} \mathrm{C}$. The average air exchange rate was $250 \mathrm{~m}^{3} /$ hour. By means of an ultrasonic vaporizer or a heater, defined amounts of the solvents were brought into the airflow.

Low and high concentrations of 1-octanol and isopropanol were used. For the "low exposure trials" the concentrations were in the range of the odor thresholds, $\approx 0.1 \mathrm{ppm}$ for 1 -octanol and $\approx 3-196 \mathrm{ppm}$ for isopropanol (18). During the 4 hours of these trials the concentration was kept constant. The mean concentrations amounted to 0.1 (SD 0.01) ppm for 1-octanol and 34.9 (SD 1.1) ppm for isopropanol.

For the "high-exposure trials" the concentrations were in the range of the German STEL values. According to the STEL rule for isopropanol (7) exposure peaks of $400 \mathrm{ppm}$ were permitted. The high-exposure trials used concentrations that varied within each hour from an exposure peak to a minimum and back to the peak. The exposure protocol of the experiment is shown in figure 1. Integration of the measured concentration data resulted in a time-weighted average exposure of 189.9 ppm (SD $132.9 \mathrm{ppm}$ ) for isopropanol during the high exposure. 1-Octanol is not regulated by a German MAK, and no STEL rule existed. According to its properties 1-octanol would be classified as a local irritant, and a
STEL rule comparable with that for isopropanol would be reasonable. Pilot trials revealed that $50 \mathrm{ppm}$ of 1 -octanol, recommended as the workplace environmental exposure limit (WEEL) (11), would be unreasonable for human volunteers. With consideration of these facts and the limited knowledge on the exposure effects on humans, a conservative average concentration of $5 \mathrm{ppm}$ (peaks $10 \mathrm{ppm}$ ) was chosen; the actual concentration data measured during the high exposure trials showed a mean exposure level of 6.4 (SD 5.3) ppm for 1-octanol.

In a crossover design each subject was exposed to the four conditions. Four subjects were exposed simultaneously, two chemically sensitive subjects and two controls. Thus half of the chemically sensitive subjects and the controls were exposed to 1-octanol first, the other half to isopropanol. Successive sessions of the participants were separated by at least 2 days. At the end of the experiment they were paid a compensation of DEM 450 ( $\approx$ USD 230).

\section{Neurobehavioral tests}

A comprehensive scaling procedure (19) was used to assess three dimensions of well-being, namely, "tenseness" (relaxed versus stressed), "tiredness" (awake versus tired), and "annoyance" (not annoying versus very annoying). Together with 7-point visual rating scales, the key words of the dimensions were presented on the computer screen. Ratings were given by means of a numerical pad on a response panel (range $1-7)$.

Acute health symptoms were assessed with an extended version of the test called Acute Symptoms from the Swedish Performance Evaluation System (SPES) $(20,21)$. The initial SPES version of 17 acute symptoms

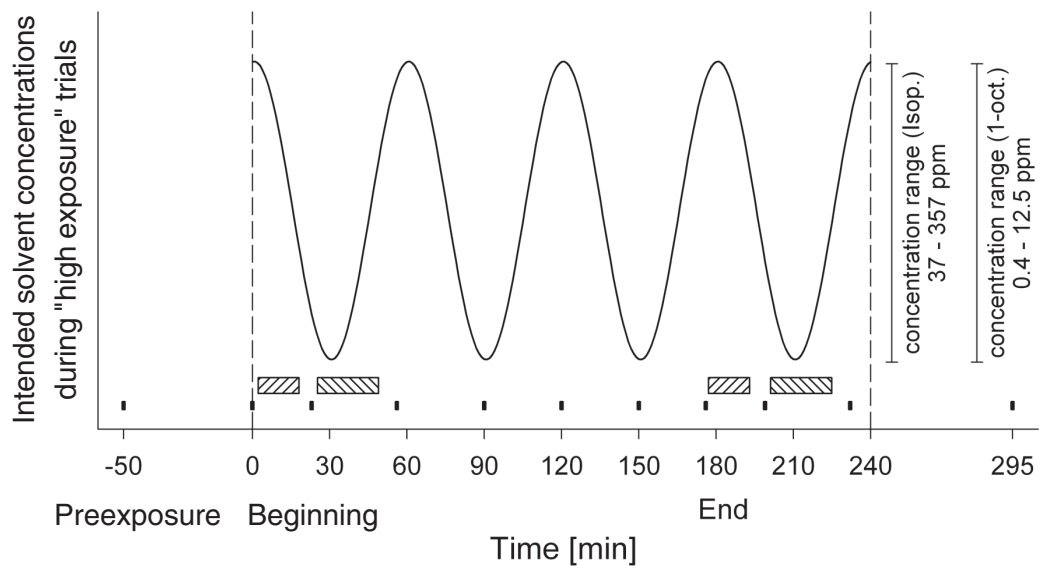

- Ratings of acute symptoms/ well-being

EIJ Performance test 1 (PT1) CRT and DA

WIIV Performance test 2 (PT2) VT
Figure 1. Illustration of the intended time courses of the solvent concentrations during the "high" exposures and the schedule of the neurobehavioral testing and symptom and annoyance ratings. (Isop = isopropanol, 1-oct $=1$-octanol, $\mathrm{CRT}=$ choice reaction time, $\mathrm{DA}=$ divided attention, VT = vigilance task) 
(eg, nausea, headache) was extended by 12 symptoms related to the olfactory system, nasal irritation, and eye irritation. Thus 29 acute symptoms were presented on the computer screen one after another, and their severities were rated on a 6-point rating scale ranging from 0 (not at all) to 5 (very, very much). Ratings of similar symptoms (eg, stink, unpleasant odor) were combined and their respective means were calculated (range 0-5). These were prenarcotic symptoms (4 symptoms), olfactory symptoms (4 symptoms), symptoms of bad taste (3 symptoms), respiratory symptoms (3 symptoms), nasal irritation (5 symptoms), eye irritation (7 symptoms), and other irritation (3 symptoms). For our study nasal and eye irritation were combined into a score of sensory irritation. The ratings of both well-being and acute symptoms were expressed as the percentage of their maximum scale value (range $0-100 \%$ ) in order to illustrate the magnitude of the ratings.

Three neuropsychological tests, which tap different cognitive functions, were used. In the Choice Reaction Time test of the SPES (20) subjects had to respond to the shape of a visual stimulus by way of moving from a starting position in the center of the response panel to one of four crosswise arranged targets. A trial of this test lasted 10 minutes. In the "divided attention" task of the German attentional test battery (TAP) (22) the subjects monitored parallel streams of stimuli presented visually and auditorily and responded to stimuli with particular characteristics by pressing a response button. The critical stimuli were equal successive tones in a sequence of high and low tones and squares in a sequence of randomly varying visual patterns. Both tasks (visual and auditory) had to be performed with the same accuracy. The task duration was approximately 8 minutes. Finally, a vigilance task in the format of a Mackworth clock test (23) was used. In the center of the computer screen, a circle of 24 red dots was displayed. Every 1000 milliseconds a yellow dot moved clockwise to the next dot. The subjects were instructed to respond by pressing a button if the yellow dot made a double step across two red dots ("double jump", $\mathrm{P}=0.05$ ) and to ignore "single jumps" ( $\mathrm{P}=0.95)$. The vigilance task lasted $30 \mathrm{~min}-$ utes.

The tests were presented on a 15 -inch color computer screen. The performance measures were reaction times, detection rates, and, where measurable, false alarms.

All the neurobehavioral tests were presented according to a fixed time schedule (see figure 1). The ratings of well-being and acute symptoms were given before $(1 \times)$, during $(9 \times)$, and after $(1 \times)$ the exposure period. The performance measures were done in two blocks [performance test 1 (PT1) and performance test 2 (PT2); see figure 1] and presented at both the beginning and the end of the exposure period.

\section{Data analysis}

Data were analyzed with SPSS 10.0 (24). Well-being and acute symptoms were assessed on ordinal scales; therefore nonparametric tests were used. For this analysis, the three ratings of well-being and acute symptoms given during the first hour of the exposure period were averaged [beginning (ie, after 1, 23 and 56 minutes)], as were those given during the last hour [end (ie, after 176, 199, and 232 minutes)]. (See figure 1.) The exposure-related effects within the four conditions were tested with Wilcoxon signed-rank tests. Separately for each condition, the preexposure score, obtained 50 minutes before the onset of exposure, was compared (i) with the mean score of the first hour and (ii) with the mean score of the last hour. The ratings obtained after 90, 120, and 150 minutes were not analyzed in this study.

Dose-related effects, that is, the differences between the high and low concentrations of the substances, were tested with Wilcoxon signed-rank tests applied to baseline-corrected ratings. These baseline-corrected ratings (difference scores) were calculated by subtracting the preexposure scores from the scores given at the beginning and the end of the exposure period. The two baseline-corrected ratings of the low concentration of each substance were compared with those calculated for the high concentration.

Mann-Whitney-U tests were used to test the effect of chemical sensitivity. The baseline-corrected ratings of the chemically sensitive subjects were compared with those of the controls for all the exposure conditions.

The performance tests were analyzed by multivariate analyses of variance (MANOVA) testing $2 \times 2 \times 2 \times 2(\times 5)$-factorial models. The first factor was the between-subject factor sensitivity, and the next three factors were the within-subject factors "substance", "concentration", and "duration of exposure" (beginning versus end). To analyze the vigilance decrement in the vigilance task, the time on the task was subdivided into five 6-minute intervals forming the "time on task" factor (given in parentheses) exclusively used for this particular test. Where indicated by the multivariate results, univariate analyses were calculated to identify the primarily affected target parameter. A significance level of $\alpha=0.05$ was chosen to test the null hypothesis of no differences between the population means. Where required, the alpha level was adjusted according to Bonferoni correction (25).

\section{Results}

\section{Well-being and acute symptoms - exposure-related effects (during the exposure session)}

Bad taste, respiratory symptoms, and other irritations were not reported during the exposures and therefore 
were excluded from the analyses. None of the four exposures provoked feelings of tenseness or prenarcotic symptoms. Table 1 shows the medians, the $90 \%$ confidence intervals $(90 \% \mathrm{CI})$, and the results of the nonparametric tests of the remaining dimensions of well-being (tiredness and annoyance), the olfactory symptoms, and sensory irritation for the three analyzed assessments.

Compared with the preexposure ratings, elevated tiredness was reported at the beginning of the low 1octanol exposure, the end of both 1-octanol exposures, and the end of the high isopropanol exposure. Among these elevated tiredness ratings, the Wilcoxon signedrank test revealed only one significant difference. Not apparent from the medians, two other comparisons were significant. Due to the specific distributions of the negative and positive sums of ranks, the Wilcoxon signedrank test revealed significant rating differences at the end of the low isopropanol exposure and at the beginning of the high 1-octanol exposure. In both cases the tiredness ratings were not elevated numerically. Altogether, none of the observed medians exceeded a value of $33.3 \%$, representing one-third of the scale range (0$100 \%)$. The confidence intervals indicated a strong variation in the tiredness rating even in the preexposure assessment.

Within the investigated exposure conditions, the annoyance ratings were elevated at both assessments of all the exposures. Except for the high isopropanol condition, the annoyance ratings remained nearly constant across the exposure periods. The confidence intervals of the annoyance ratings indicated strong interindividual differences. While some subjects did not feel annoyed by the exposure, the ratings of others indicated that the exposure was very annoying. Only for high 1-octanol exposure was the lower limit of the confidence interval greater than zero, indicating some feelings of annoyance for all the subjects. However, at no time was the median of the annoyance ratings in the highest one-third of the scale range.

Olfactory symptoms were reported during both 1octanol exposures and during the high isopropanol exposure. Reports of sensory irritation were elevated exclusively during the high 1-octanol exposure. With respect to the general level of the reported symptoms, the results showed that all the median symptom scores were lower than $50 \%$ of the scale range. The confidence intervals of the olfactory symptoms showed that, individually, both high and low ratings were given throughout the 1-octanol exposures and the high isopropanol exposure. From the beginning to the end of the exposures, the symptom scores showed no marked increase. In fact, table 1 shows that the olfactory symptoms decreased from the beginning to the end during all the exposures.

\section{Well-being and acute symptoms — dose-related effects (within-substance)}

The results of the dose-response analysis are given in table 2. Neither at the beginning (first hour) nor at the

Table 1. Descriptive statistics of the ratings for tiredness, annoyance, olfactory symptoms and sensory irritation and the Wilcoxon test results comparing the preexposure ratings with those obtained at the beginning or end of the exposure periods. $[90 \% \mathrm{Cl}=90 \% \mathrm{confi}-$ dence interval, $\mathrm{T}$ = sum of tested ranks, $\mathrm{n}=$ number of pairs (nonzero differences), $\mathrm{P}$ = exact $\mathrm{P}$-value of the Wilcoxon test]

\begin{tabular}{|c|c|c|c|c|c|c|c|c|c|c|c|c|}
\hline & \multicolumn{2}{|c|}{ Preexposure } & \multicolumn{2}{|c|}{ Beginning } & \multicolumn{2}{|c|}{ End } & \multicolumn{3}{|c|}{ Beginning versus preexposure } & \multicolumn{3}{|c|}{ End versus preexposure } \\
\hline & Median & $90 \% \mathrm{Cl}$ & Median & $90 \% \mathrm{Cl}$ & Median & $90 \% \mathrm{Cl}$ & $\mathrm{T}$ & $\mathrm{n}$ & $\mathrm{P}$ & $\mathrm{T}$ & $n$ & $P$ \\
\hline \multicolumn{13}{|l|}{ Tiredness } \\
\hline Isopropanol (low) & 16.7 & $0.0-50.0$ & 16.7 & $0.0-66.7$ & 16.7 & $0.0-66.7$ & 41 & 17 & 0.063 & 33 & 19 & $0.007^{\text {a }}$ \\
\hline 1-Octanol (low) & 16.7 & $0.0-66.7$ & 25.0 & $0.0-50.0$ & 33.3 & $0.0-66.7$ & 93 & 20 & 0.563 & 47 & 21 & 0.009 \\
\hline Isopropanol (high) & 16.7 & $0.0-50.0$ & 16.7 & $0.0-66.7$ & 33.3 & $0.0-83.3$ & 29 & 15 & 0.069 & 16 & 19 & $0.001^{\text {a }}$ \\
\hline 1-Octanol (high) & 16.7 & $0.0-66.7$ & 16.7 & $0.0-66.7$ & 25.0 & $0.0-83.3$ & 16 & 16 & $0.003^{a}$ & 39 & 19 & 0.022 \\
\hline \multicolumn{13}{|l|}{ Annoyance } \\
\hline Isopropanol (low) & 0.0 & $0.0-33.3$ & 8.3 & $0.0-33.3$ & 8.3 & $0.0-33.3$ & 3 & 12 & $0.004^{a}$ & 5 & 12 & 0.004 a \\
\hline 1-0ctanol (low) & 0.0 & $0.0-35.0$ & 16.7 & $0.0-75.8$ & 16.7 & $0.0-59.2$ & 0 & 14 & $0.001^{\mathrm{a}}$ & 0 & 17 & $0.001^{\mathrm{a}}$ \\
\hline Isopropanol (high) & 0.0 & $0.0-33.3$ & 16.7 & $0.0-75.8$ & 33.3 & $0.0-59.2$ & 3 & 19 & $0.001^{\mathrm{a}}$ & 0 & 20 & $0.001^{\mathrm{a}}$ \\
\hline 1-Octanol (high) & 0.0 & $0.0-59.2$ & 33.3 & $7.5-83.3$ & 33.3 & $7.5-83.3$ & 3 & 23 & $0.001^{\mathrm{a}}$ & 6 & 23 & $0.001^{\text {a }}$ \\
\hline \multicolumn{13}{|l|}{ Olfactory symptoms } \\
\hline Isopropanol (low) & 0.0 & $0.0-28.3$ & 2.5 & $0.0-20.0$ & 0.0 & $0.0-17.8$ & 26 & 14 & 0.095 & 22 & 10 & 0.584 \\
\hline 1-Octanol (low) & 0.0 & $0.0-39.3$ & 15.0 & $0.0-70.5$ & 10.0 & $0.0-55.5$ & 7 & 18 & $0.001^{\mathrm{a}}$ & 11 & 15 & $0.003^{a}$ \\
\hline Isopropanol (high) & 0.0 & $0.0-43.8$ & 25.0 & $0.0-90.5$ & 15.0 & $0.0-47.8$ & 2 & 20 & $0.001^{a}$ & 24 & 19 & $0.003^{a}$ \\
\hline 1-Octanol (high) & 0.0 & $0.0-70.5$ & 42.5 & $0.0-85.5$ & 30.0 & $0.0-60.0$ & 0 & 21 & $0.001^{\mathrm{a}}$ & 14 & 20 & $0.001^{\text {a }}$ \\
\hline \multicolumn{13}{|l|}{ Sensory irritation } \\
\hline Isopropanol (low) & 0.9 & $0.0-20.0$ & 0.0 & $0.0-22.0$ & 0.9 & $0.0-23.0$ & 14 & 7 & 1.000 & 36 & 12 & 0.652 \\
\hline 1-0ctanol (low) & 0.0 & $0.0-22.0$ & 1.8 & $0.0-34.6$ & 1.8 & $0.0-25.7$ & 14 & 13 & 0.025 & 31 & 15 & 0.162 \\
\hline Isopropanol (high) & 0.9 & $0.0-19.2$ & 3.6 & $0.0-22.8$ & 4.5 & $0.0-26.6$ & 42 & 17 & 0.168 & 24 & 14 & 0.113 \\
\hline 1-Octanol (high) & 0.0 & $0.0-21.0$ & 11.0 & $0.0-38.4$ & 7.2 & $0.0-34.8$ & 0 & 18 & $0.001^{\mathrm{a}}$ & 0 & 18 & $0.001^{\mathrm{a}}$ \\
\hline
\end{tabular}

a Significant according to the Bonferoni-corrected $\alpha$ of $\alpha=0.05(P<0.0083)$. 
Table 2. Descriptive statistics of the baseline-corrected ratings for tiredness, annoyance, olfactory symptoms and sensory irritation and the Wilcoxon test results comparing the high and low concentrations of the substances. $(90 \% \mathrm{Cl}=90 \%$ confidence interval, $\mathrm{T}=$ sum of tested ranks, $n=$ number of pairs (nonzero differences), $\mathrm{P}=$ exact $\mathrm{P}$-value of the Wilcoxon test)

\begin{tabular}{|c|c|c|c|c|c|c|c|c|c|c|}
\hline & \multicolumn{5}{|c|}{ Beginning versus preexposure } & \multicolumn{5}{|c|}{ End versus preexposure } \\
\hline & \multicolumn{2}{|c|}{ Median } & \multicolumn{3}{|c|}{ High versus low } & \multicolumn{2}{|c|}{ Median } & \multicolumn{3}{|c|}{ High versus low } \\
\hline & Low & High & $\mathrm{T}$ & $\mathrm{n}$ & $P$ & Low & High & $\mathrm{T}$ & $\mathrm{n}$ & $P$ \\
\hline \multicolumn{11}{|l|}{ Tiredness } \\
\hline Isopropanol & 0.0 & 0.0 & 60 & 15 & 0.988 & 0.0 & 16.7 & 45 & 17 & 0.202 \\
\hline 1-Octanol & 0.0 & 0.0 & 50 & 16 & 0.315 & 16.7 & 0.0 & 82 & 19 & 0.601 \\
\hline \multicolumn{11}{|l|}{ Annoyance } \\
\hline Isopropanol & 0.0 & 16.7 & 22 & 15 & 0.026 & 0.0 & 16.7 & 17 & 18 & $0.002^{a}$ \\
\hline 1-Octanol & 16.7 & 16.7 & 75 & 18 & 0.693 & 16.7 & 16.7 & 71 & 18 & 0.527 \\
\hline \multicolumn{11}{|c|}{ Olfactory symptoms } \\
\hline Isopropanol & 0.0 & 20.0 & 7 & 21 & $0.001^{a}$ & 0.0 & 10.0 & 34 & 22 & $0.002^{\mathrm{a}}$ \\
\hline 1-Octanol & 10.0 & 35.0 & 41 & 20 & 0.015 & 2.5 & 17.5 & 48 & 21 & 0.016 \\
\hline \multicolumn{11}{|l|}{ Sensory irrition } \\
\hline Isopropanol & 0.0 & 0.9 & 42 & 18 & 0.057 & 0.0 & 0.0 & 27 & 16 & 0.033 \\
\hline 1-Octanol & 0.0 & 10.0 & 9 & 20 & $0.001^{\text {a }}$ & 0.0 & 7.2 & 9 & 17 & $0.001^{\mathrm{a}}$ \\
\hline
\end{tabular}

a Significant according to the Bonferoni-corrected $\alpha$ of $\alpha=0.05(P<0.0125)$.

end (last hour) of the exposures did the Wilcoxon tests reveal a dose-dependent increase in the tiredness ratings. For both assessments increments of annoyance over the preexposure levels were stronger with the high isopropanol exposure than with the low-level exposure, but this difference was significant only at the end of the exposure period. Table 2 shows that, on the average, the annoyance increments during both assessments of the 1-octanol exposures were comparable.

The difference scores of the olfactory symptoms obtained in the first (beginning) and the last (end) hour of the high isopropanol exposure were significantly higher than those observed during the low isopropanol exposure. During the high 1-octanol exposures the increase in the olfactory symptoms was more pronounced than during the low exposure; however, this occurrence did not quite reach significance. Throughout both high exposures, the increment of the olfactory symptoms diminished from the beginning to the end of the exposure period. 1-Octanol provoked a stronger increase in sensory irritation during the high exposure.

\section{Well-being and acute symptoms - sensitivity-related effects (between-subject)}

None of the difference scores of the two symptom areas (olfaction and sensory irritation) given in table 2 were affected by the between-subject factor "chemical sensitivity". During the 1-octanol exposures the between-subject factor "chemical sensitivity" showed a strong impact on the annoyance ratings. At the beginning of the low 1-octanol exposure the difference scores of the annoyance ratings of the chemically sensitive subjects (median 25.0, 90\% CI 0.0-83.3) were higher than those of the controls (median 0.0, 90\% CI 0.0-66.8). In the course of the 4 hours of the exposure this difference vanished, and at the end both groups yielded medians of 16.7. Comparable effects occurred for the annoyance ratings given during high 1-octanol exposure. The annoyance reporting of the chemically sensitive subjects (beginning: median 25.0, 90\% CI 0.0-50.0; end: median $25.0,90 \%$ CI $0.0-50.0$ ) and the controls (beginning: median 16.7, 90\% CI 0.0-66.7; end: median 16.7, 90\% CI 0.0-50.0) differed. These differences were temporally stable and significant at both times. During the isopropanol exposures the annoyance reporting of the chemically sensitive subjects was slightly higher, but none of the calculated comparisons yielded statistical significance. Since table 2 showed no pronounced increase in tiredness during nearly all the exposures, these ratings were not analyzed with respect to the betweensubject effects.

\section{Performance measurements}

The analysis of variance of the "choice reaction time" task did not yield any significant exposure-related within-subject effect or any between-subject effects.

Regardless of the exposure conditions, the analysis of the vigilance task revealed a main effect $\left[\mathrm{F}_{(8,184)}=8.7\right.$, $\mathrm{P}<0.01]$ of the factor "time on task". Increasing reaction times and decreasing detection rates across the 30 minutes of the vigilance task were observed throughout all the exposures. On the average, the detection rates showed an almost linear decline from $90 \%$ to $83 \%$ across the 30 minutes $\left[\mathrm{F}_{(4)}=20.5, \mathrm{P}<0.01\right]$ of the vigilance task. Simultaneously, the reaction times increased significantly $\left[\mathrm{F}_{(4)}=8.2, \mathrm{P}<0.01\right]$ from 520 to 557 
milliseconds, following a quadratic function. The analysis of variance yielded no further between-subject, within-subject, or interaction effects.

The performance measures of the divided attention task were affected by the substance and the interaction of sensitivity and substance. The multivariate analysis revealed that, regardless of the level of exposure, the performance during the 1-octanol exposures differed from that during the isopropanol exposures $\left[\mathrm{F}_{(3,18)}=4.5\right.$, $\mathrm{P}=0.02]$. In addition to this main effect, an interaction between the two factors substance and chemical sensitivity $\left[\mathrm{F}_{(3,18)}=3.6, \mathrm{P}=0.03\right]$ indicated different performance patterns for the chemically sensitive subjects and the controls. Univariate analyses showed that primarily the detection rates were affected. Figure 2 illustrates the main effect of substance and interaction.

The left side of figure 2 clearly shows that, during exposure to 1-octanol, the detection rates of the divided attention task were lower than during exposure to isopropanol. The right side of figure 2 shows that this substance-related difference was caused by the lower detection rates of the chemically sensitive subjects during both 1-octanol exposures. The other target parameters of the divided attention task were not affected by 1-octanol.

\section{Discussion}

Consistent with previous reports $(2,3)$, our study confirmed that short-term exposures to isopropanol at TLV concentrations provoke only subjective effects, as indicated by slightly enhanced ratings of olfactory symptoms and annoyance. Confirming the study of Sethre et al (2), we showed that performance was not affected in neurobehavioral tests. Even weak reports of sensory irritations were not reported, and a time-weighted average exposure of $200 \mathrm{ppm}$ appeared to be insufficient to provoke reports of sensory irritation. A recent study (26) revealed that experimental exposure to $400 \mathrm{ppm}$ of isopropanol was necessary to excite weak ratings of sensory irritation. The tiredness ratings at the end of both isopropanol exposures differed from those given before the exposure period. This result was probably a simple consequence of the duration of the experiment, because there was no dose-dependency and numerically comparable increases were observed for the 1-octanol exposures. Thus neither the tiredness ratings nor the prenarcotic symptoms indicated sedative effects of isopropanol.

In summary, for the effects of 1-octanol, it was shown that exposure even to a low concentration was associated with enhanced olfactory symptoms, enhanced annoyance, and partly reduced performance in one of

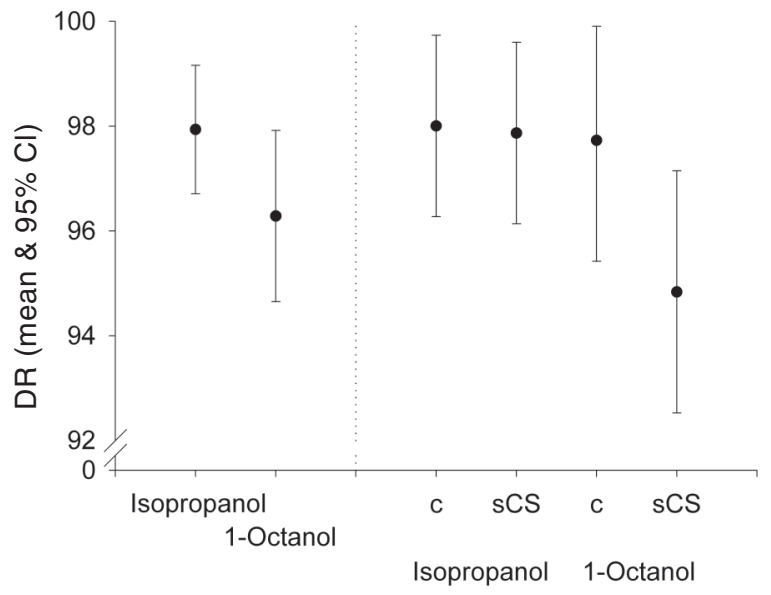

Figure 2. Mean detection rates (DR) for the "divided attention" task during the isopropanol and 1-octanol exposure in general and also separated for the sensitivity groups. $(95 \% \mathrm{Cl}=95 \%$ confidence interval, $\mathrm{c}=$ controls, $\mathrm{sCS}=$ chemically sensitive subjects)

the neurobehavioral tasks. Furthermore, exposure to 6.4 ppm of 1-octanol excited weak signs of sensory irritation, and this effect showed a clear dose-dependency. Nevertheless, most of the observed effects showed no clear dose-response relation, but occurred even during low-level exposures to 1-octanol or exclusively in the group of sensitive subjects. Especially the fact that the performance of the chemically sensitive subjects was slightly reduced during both 1-octanol exposures is implausible.

Physiologically, a de-arousing mechanism of acute solvent exposure is reasonable $(27,28)$. This mechanism would suggest that (i) the detection rate should be more strongly reduced during high exposures, (ii) other target parameters of the task (eg, reaction times) should be affected simultaneously, (iii) other attentional functions (eg, vigilance) should be influenced, and (iv) chemical sensitivity should not interact with behavioral measures indicating prenarcotic effects. The obtained results for 1-octanol are in contrast to this mechanism, and therefore, the interpretation is difficult. It can be hypothesized that chemosensory stimulus processing in association with exposure to 1-octanol may explain this isolated neurobehavioral effect.

Dick \& Ahlers (29) argued that even feelings of sensory irritation should be considered carefully in the process of regulatory "limit setting", since they may be distracting and interfere with job performance and safety. Such a behavioral consequence of chemosensory stimulation was only partly observable for the chemically sensitive subjects during the 1-octanol exposures, but the proposed distractive mechanism should affect all subjects equally.

More theoretically, neuropsychological models of attention provide a framework with which to explain 
distractive effects of chemosensory stimulation. Norman \& Shallice (30) developed a model of attention that is composed of a multitude of subsystems of perceptual and cognitive processing, which are monitored by a "supervisory attentional system". This system is needed in situations that are judged difficult, dangerous, or, in any case, nonroutine. In chemically sensitive subjects the perception of 1-octanol may activate the supervisory attentional system, and target detection may be disturbed in the divided attention task. Nevertheless, theoretically this reasoning may apply to the data, but, on the other hand, it is more plausible that this minor effect represents a nonspecific annoyance effect observed in sensitive people only.

Chemical sensitivity showed no impact on subjective symptoms and annoyance during the isopropanol exposures. Only the combination of 1-octanol and chemical sensitivity reinforced the ratings of annoyance. Again the chemosensory properties of 1-octanol seemed to be crucial. Two major distinctions between 1-octanol and isopropanol are obvious. Depending on procedural details, the odor threshold of isopropanol (3.14-196 ppm) is 25-1500 times higher than that of 1-octanol ( $\approx 0.13 \mathrm{ppm})$ (18). In addition, ComettoMuniz et al (12) compared propanol and 1-octanol and showed that the perceived intensity of the two substances differs markedly at suprathreshold concentrations. Propanol had a less intense odor than 1-octanol. Due to the similarity with propanol comparable properties should apply to isopropanol. This discrepancy between isopropanol (high odor threshold, subdued odor intensity) and 1-octanol (low odor threshold, intense odor) may explain the lack of dose-dependency observed for the annoyance and olfactory symptom ratings in response to 1-octanol.

Secondary effects of these sensory characteristics possibly account for the interindividual differences observed for the annoyance ratings. Differences in sensory processing are unlikely, because similar odor thresholds were measured for the two groups of subjects. The cognitive stages of chemosensory stimulus processing might differ however. The manifestation of chemical sensitivity is associated with the experience of adverse health effects (eg, nausea) in response to chemicals or chemical odors. According to these experiences, an altered cognitive feedback loop is conceivable for chemically sensitive subjects. After the sensation of 1-octanol, an additional negative affective component (9), solely occurring in the chemically sensitive subjects, may aggravate the feelings of annoyance. The impact of such cognitive factors on annoyance ratings after acute solvent exposure has been shown by Dalton et al (31). It seems plausible to assume such an alteration, but the design of our study was not suitable for testing the hypothesis of an altered feedback loop of chemo- sensory stimuli processing in sensitive subjects more precisely.

Sensory symptoms are a critical endpoint for TLV setting (29), is and the reported sensory irritation during "high" 1-octanol exposure is relevant in this respect. Even though the absolute level of the subjective ratings of sensory irritation was low (10\% and $7.2 \%$ of the scale range), this finding must be evaluated against the fact that the applied "high" concentration of 1-octanol was low $(12.8 \%$ of the WEEL and $\approx 8 \%$ of the NPT). Within the same group of subjects and with the same scaling method, exposure to isopropanol at TLV concentrations did not provoke reports of sensory irritations at all. Nevertheless, the evaluation of this result is difficult. Subjective data indicates that the distinction between olfactory and trigeminal (sensory irritation) stimulation is difficult (9). Therefore, ratings of sensory irritation may be affected by the strong olfactory stimulation caused even by low-level exposure to 1-octanol. Without the integration of the physiological indicators of sensory irritation, it is difficult (i) to distinguish between annoying odors and annoying irritation and (ii) to benchmark the strength of reported sensory irritation with respect to adversity. Thus, with respect to external validation and benchmarking, our study has some shortcomings, and further experimental studies need to pay more attention to the relations among SPES questionnaire scores, annoyance ratings, and physiological indicators of sensory irritation.

Nevertheless, the results of our study suggest that the existing WEEL (50 ppm) published by the American Industrial Hygiene Association might be insufficient to protect exposed workers from sensory irritation. Additional studies are needed to explore the distractive effects of chemosensory stimulation during demanding performance tasks.

\section{Acknowledgments}

The German Research Council/Foundation (Deutsche Forschungsgemeinschaft) supported the study with grant SE 535/ 5-1.

Thanks are due to the staff of the neurobehavioral toxicology, analytical chemistry, and clinical occupational medicine units for their hard work during the experiments.

\section{References}

1. Litovitz T. The alcohols: ethanol, methanol, isopropanol, ethylene glycol. Pediatr Clin North Am 1986;33(2):311-23. 
2. Sethre T, Läubli T, Berode M, Krueger H. Neurobehavioural effects of experimental isopropanol exposure. Int Arch Occup Environ Health 2000;73:105-12.

3. Muttray A, Jung D, Rose DM, Hommel G, Konietzko J. Acute effects of exposure to $360 \mathrm{ppm}$ Isopropanol on healthy man. Centr Eur J Occup Environ Med 1998;4:288-93.

4. Triebig G, Fritz M, Schaller KH, Helbing F, Bünte EM, Kufner K, et al. Arbeitsmedizinische Untersuchungen bei beruflich Iso-Propanol-exponierten Frauen. [Medical investigation of women occupationally exposed to isopropanol.] Arbeitsmed Sozialmed Präventivmed 1989;24:27-31.

5. Sethre T, Läubli T, Riediker M, Hangartner M, Krueger H. Neurobehavioral effects of low level solvent exposures in a foundry. Centr Eur J Occup Environ Med 1998;4:316-27.

6. Maizlish NA, Langolf GD, Whitehead LW, Fine LJ, Albers JW, Goldberg J, et al. Behavioural evaluation of workers exposed to mixtures of organic solvents. Br J Ind Med 1985; 42:579-90.

7. Deutsche Forschungsgemeinschaft (DFG). List of MAK and BAT values 2000: maximum concentrations and biological tolerance values at the workplace. Weinheim (Germany): Wiley-VCH; 2000

8. American Conference of Governmental Industrial Hygienists (ACGIH). 2000 TLVs and BEIs: threshold limit values for chemical substances and physical agents biological exposure indices. Cincinnati $(\mathrm{OH})$ : ACGIH Worldwide; 2000.

9. Dalton PH, Dilks DD, Banton MI. Evaluation of odor and sensory irritation thresholds of methyl isobutyl ketone in humans. Am Ind Hyg Assoc J 2000;61:340-50

10. Deutsche Forschungsgemeinschaft (DFG). Gesundheitsschädliche Arbeitsstoffe. Toxikologisch-arbeitsmedizinische Begründung von MAK-Werten: 2-ethylhexanol. MAK, 30. Lieferung. Weinheim (Germany): Wiley-VCH; 2000.

11. American Industrial Hygienic Association (AIHA). Workplace environmental exposure level [WEEL] 1-octanol. Fairfax (VA): AIHA Press; 1992.

12. Cometto-Muniz JE, Cain WS. Thresholds for odor and nasal pungency. Physiol Behav 1990;48:719-25.

13. Cometto-Muniz JE, Cain WS. Trigeminal and olfactory sensitivity: comparison of modalities and methods of measurement. Int Arch Occup Environ Health 1998;71:105-10.

14. Cometto-Muniz JE, Cain WS. Efficacy of volatile organic compounds in evoking nasal pungency and odor. Arch Environ Health 1993;48:309-14.

15. Fang Z, Ionescu P, Chortkoff BS, Kandel L, Sonner J, Laster MJ, et al. Anesthetic potencies of n-alkanols: results of additivity and solubility studies suggest a mechanism of action similar to that for conventional inhaled anesthetics. Anesth Analg 1997;84:1042-8.

16. Bell IR, Miller CS, Schwartz GE, Peterson JM, Amend D. Neuropsychiatric and somatic characteristics of young adults with and without self-reported chemical odor intolerance and chemical sensitivity. Arch Environ Health 1996;51:9-21.

17. Kiesswetter E, Sietmann B, Zupanic M, van Thriel C, Golka K, Seeber A. Verhaltenstoxische Aspekte der Prävalenz und Ätiologie "multipler chemischer Sensitivität" [Neurobehavioral aspects of the prevalence and etiology of "multiple chemical sensitivity"]. Allergologie 1999;22:719-35.

18. Ruth JH. Odor threshold and irritation levels of several chemical substances: a review. Am Ind Hyg Assoc J 1986;47:14251.

19. Seeber A, Blaszkewicz M, Golka K, Kiesswetter E. Solvent exposure and ratings of well-being: dose-effect relationships and consistency of data. Environ Res 1997;73:81-91.

20. Gamberale F, Iregren A, Kjellberg A. Computerized performance testing in neurotoxicology. Why, what, how, and whereto? The SPES example. In: Russel RW, Flattau PE, Pope AM, editors. Behavioral measures of neurotoxicity. Washington (DC): National Academy Press; 1990. p 359.

21. Iregren A. Computer-assisted testing. In: Costa LG, Manzo L, editors. Occupational neurotoxicology. Boca Raton (FL): CRC Press; 1998. p 213-31.

22. Zimmermann P, Fimm B. Testbatterie zur Aufmerksamkeitsprüfung (TAP) Version 1.0. Würselen: PSYTEST; 1993.

23. Parasuraman R, Warm JS, See JE. Brain systems of vigilance. In: Parasuraman R, editor. The attentive Brain. Cambridge (MA): MIT Press; 2000. p 221-56.

24. SPSS Inc. SPSS 10.0 Syntax Reference Guide. Chicago (IL): SPSS Inc; 1999.

25. Bortz J, Lienert GA, Boehnke K. Verteilungsfreie Methoden in der Biostatistik. Berlin: Springer-Verlag; 1990.

26. Smeets MA, Mauté C, Dalton PH. Acute sensory irritations from exposure to isopropanol (2-propanol) at TLV in workers and controls: objective versus subjective effects. Ann Occup Hyg 2002;46:359-73.

27. Dick RB. Short duration exposures to organic solvents: The relationship between neurobehavioral test results and other indicators. Neurotoxicol Teratol 1988;10:39-50.

28. Muttray A, Kurten R, Jung D, Schicketanz KH, Mayer-Popken O, Konietzko J. Acute effects of 200 ppm 1,1,1-trichloroethane on the human EEG. Eur J Med Res 2000;5:375-84.

29. Dick RB, Ahlers H. Chemicals in the workplace: incorporating human neurobehavioral testing into the regulatory process. Am J Ind Med 1998;33:439-53.

30. Norman DA, Shallice T. Attention to action: willed and automatic control of behavior. In: Davidson RJ, Schwartz GE, Shapiro D, editors. Consciousness and self-regulation. New York (NY): Plenum Press; 986. p 1-18.

31. Dalton P, Wysocki CJ, Brody MJ, Lawley HJ. The influence of cognitive bias on the perceived odor, irritation and health symptoms from chemical exposure. Int Arch Occup Environ Health 1997;69:407-17.

Received for publication: 8 July 2002 